\title{
About the class of approximation by generalized characters
}

\author{
Dang Van Vinh*
}

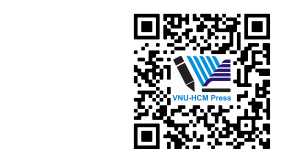

Use your smartphone to scan this QR code and download this article

Ho Chi Minh City University of Technology, VNU-HCM, Vietnam

\section{Correspondence}

Dang Van Vinh, Ho Chi Minh City University of Technology, VNU-HCM, Vietnam

Email: dangvvinh@hcmut.edu.vn

History

- Received: 4-12-2019

- Accepted: 17-12-2020

- Published: 31-12-2020

DOI : 10.32508/stdjet.v3iSI3.635

\section{Check for updates}

Copyright

(c) VNU-HCM Press. This is an openaccess article distributed under the terms of the Creative Commons Attribution 4.0 International license.

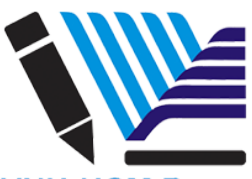

VNU-HCM Press

\section{ABSTRACT}

The common concept of approximation of algebraic system was given in the research of Russian academician Mal'cev A.I. In this article, Mal'cev showed a connection between the finitely approximation of algebraic system with respect to a given predicate and the problem of solvability of this predicate in the system. The notion of a finitely approximable semigroup is also mentioned. Some results about the approximation of semigroup were proposed by A. I. Mal'cev in the article. The research interest in an approximation of algebraic structures is inspired by Professor M.M. Lesokhin's work. There exist three main problems of approximation of algebraic structures. The first is finding conditions for the approximation of a structure $A$ to a given structure $B$ with respect to predicates using special kind of mappings from $A$ to $B$, the second is finding a minimal approximated structure for a certain set of algebraic structures and the last is finding a class of approximated algebraic structures for a specified set of structures. Our research is to further investigate the problem of finding a class of approximation for a certain class of semigroups with respect to various predicates. In this research, we pay our attention to an important type of mappings that are generalized characters. Given a certain class $K_{1}$ of algebraic structures. We study a problem of finding a class $K_{2}$ of algebraic structures such that the class $K_{1}$ is approximable into $K_{2}$ with respect to various predicates by generalized characters from $K_{1}$ to $K_{2}$. The problem of minimization of approximation is also considered. Some theorems related to the problem of constructing an approximation class are obtained. The problem in question is much more complicated and actual than the approximation problem we have been studying before ${ }^{1-5}$. The results of the description of the approximation class play an important role in studying the solvability problem of the predicate $P$ in the class of semigroups K. In particular, if the approximation class consists of finite semigroups, then this problem is solved positively. Even more difficult is the problem of determining the necessary conditions that class $K_{1}$ is an approximation class for a given class $\mathrm{K}$ with respect to different predicates.

Key words: Approximation, class of approximation, minimal approximation, generalized characters

\section{INTRODUCTION}

A problem of approximation of algebraic structures with respect to various predicates has been proposed and studied by Professor M. M. Lesokhin ${ }^{6,7}$ and his students $^{1-14}$. They have found a necessary and sufficient conditions for approximation of algebraic structures with respect to many important predicates, such as "equality of two elements", "belonging of an element in a substructure", "belonging of an element in the group", "the relation of regular conjugation", "Green $\ell-, \Re-, h-, d$ - equivalency", "belonging of an element in the monogenic subsemigroup", etc. The problem of approximation of algebraic structures consists of three components. The first component is a set of algebraic structures such as groups, finite groups, semigroups, compact semigroups, fields,...; the second component is a set of important predicates; and the last component is a set of functions such as homomorphisms, continuous characters, continuous bicharacters, generalized characters, etc.
A formulation of a research of approximation is followed in this manner: we start with a structure $B$ and consider a set $\Phi$ of all mappings from a structure $A$ to the structure $B$. Then we select a predicate $P$ and try to find out necessary conditions for approximation of $A$ with respect to the predicate $P$ by mapping from $\Phi$. After that, we determine sufficient conditions for the approximation. Finally, we minimize the structure $B$ so that $A$ is still approximable into a proper substructure of the structure $B$.

In this research, we begin with an important class of structures $K$ and a predicate $P$. Then we look for a class of structures $K_{1}$, such that $K$ is approximable by generalized character from $K$ into $K_{1}$ with respect to the given predicate $P$. This problem is much more complicated and has an important role for the study of the class of structures $K$. A particular difficulty stands out when we try to discover necessary conditions for the class $K_{1}$ to be a class of approximation for the given class $K$. 


\section{PRELIMINARIES}

Definition 2.1. Let $A$ be a semigroup and $B$ be a commutative semigroup. A generalized character is a homomorphism from $A$ into the semigroup $B$.

Let $A$ and $B$ be two algebraic structures with the same type, $\Phi$ be the set of all homomorphisms from $A$ to $B$, and $P$ be a predicate defined on the set that consists of $A$, all subsets $\delta(A)$ of $A$ and all images of $A$ and $\delta(A)$ under the characters from $\Phi$.

Definition 2.2. ${ }^{2}$ An algebraic structure $A$ is said to be approximable by homomorphisms from $\Phi$ with respect to $P$, if for a pair of subsets $A_{1}, A_{2}$ from $A$ such that $P\left(A_{1}, A_{2}\right)$ is false, there exists $\varphi \in \Phi$ such that $P\left(\varphi\left(A_{1}\right), \varphi\left(A_{2}\right)\right)$ is also false.

Definition 2.3. ${ }^{1,15}$ Let $K_{1}$ and $K_{2}$ be two classes of algebraic structures with the same type and $P$ be a predicate.

The class $K_{2}$ is said to be a class of approximation with respect to the predicate $P$ for the class $K_{1}$, if every algebraic structure from $K_{1}$ is approximable into $K_{2}$ with respect to $P$.

Definition 2.4. ${ }^{1,15}$ If every structure from the class $K_{1}$ can be embedded into some structure from a class $K_{2}$, then we write $K_{1} \prec K_{2}$

It's obvious that, if $K_{1}$ is a class of approximation for the class $K$ and $K_{1} \prec K_{2}$, then $K_{2}$ is also a class of approximation for the class $K$.

Definition 2.5. ${ }^{1,15}$ Let $S$ and $K$ be two classes of algebraic structures with the same type and $P$ be a predicate.

The class $K$ is called a minimal class of approximation for the class $S$ with respect to $P$, if the following two conditions hold:

(i) The class $K$ is a class of approximation for the class $S$;

(ii) if a class $K_{1}$ is a class of approximation for $S$ and $K_{1} \prec K_{2}$, then $K \subset K_{1}$.

Definition 2.6. ${ }^{2,5}$ Let $Q$ be the set of all prime numbers. Let $G_{p}, p \in Q$ be the quasi-cyclic group of the type $p^{\infty}$ with an identity $e_{p}$ and with an operation denoted by $\otimes_{p}$.

Let us denote $C^{*}=\underset{p \in Q}{U} G_{p}$. Define in $C^{*}$ a multiplication as follows.

$\forall a_{p}, a_{q} \in C^{*}$

$a_{p} * a_{q}=\left\{\begin{array}{c}a_{p} \otimes_{p} a_{q}, \text { if } p=q ; \\ a_{\max \{p, q\}}, \text { if } p \neq q \text { and } \max \{p, q\}>3 \\ e_{5}, \text { if } p \neq q \text { and } \max \{p, q\}=3\end{array}\right.$

Direct calculation shows that $C^{*}=\left(C^{*}, *\right)$ is a semigroup.

Using $G_{p}, p \in Q$, we can create an infinite number of semigroups with different strutures. Let us denote
$C$ by the set of all these semigroups. Interesting that, every semigroup from $C$ is a minimal semigroup approximation for many classes with respect to common predicates.

Two types of semilattice: The first picture in Figure 1 illustrates the semigroup $C^{*}$. The second picture representes another semigroup formed from $G_{p}, p \in Q$.

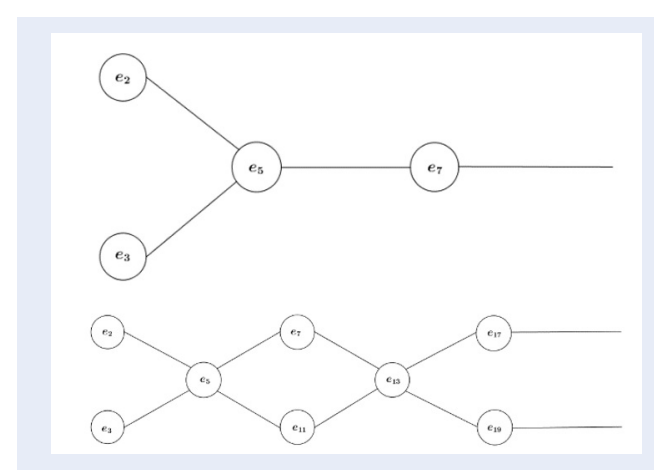

Figure 1: Two types of the semigroup $C^{*}$

Definition 2.7. Let $S$ be a semigroup and $a, b$ be two elements of the semigroup $S$.

We define:

$1 / a \mathrm{l} b$, if $a \cup S a=b \cup S b$. In other words, $S^{1} a=S^{1} b$. 2/ $a \Re b$, if $a \cup a S=b \cup b S$ or $a S^{1}=b S^{1}$.

$3 /$ The relation $l$ and $\Re$ are commute and the relation 1 o $\mathfrak{R}=\mathfrak{R}$ o 1 is denoted by $d$ relation.

4/ $h$-relation is $h=l \cap \Re$ (Clifford et al. (1977), pages 47 and 48$)^{16}$.

\section{CLASS OF APPROXIMATION}

Vinh et al. (2019) have proved that the necessary and sufficient condition for the class $S_{1}$ to be a class of approximation with respect to the predicate of equality of two elements for the class $C_{1}$ of commutative semigroups of idempotents is that the class $S_{1}$ must contain a semigroup that is isomorphic to the semigroup of two elements $B=\{0 ; 1\}$. Vinh et al. (2019) have found a necesary and sufficient condition for the class $S_{2}$ to be a class of approximation with respect to the predicate of the possible belonging of an element to a subsemigroup for the class $C_{1}$ is that the class $S_{2}$ contains a semigroup that is isomorphic to the semilattice of three elements $T=\left\{e_{1}, e_{2}, e_{3}\right\}$, where $e_{3}=e_{3} e_{1}=e_{3} e_{2}, e_{2}=e_{2} e_{1}$.

In this paper, we continue our research about the class of approximation for other classes of semigroups with respect to different predicates.

Theorem 3.1. Let $X_{1}$ be the class of commutative and separative semigroups and $Y_{1}$ be a class of semigroups. If some semigroup from the class $Y_{1}$ is isomorphic to 
the semigroup $C^{*}$, then the class $Y_{1}$ is a class of approximation for the class $X_{1}$ with respect to the predicate of equality of two elements.

Proof. Assume that $Y_{1}$ contains a semigroup $M$ that is isomorphic to the semigroup $C^{*}$.

Let $A$ be a semigroup from the class $X_{1}$.

Because $A$ is commutative, (see the book of Clifford et al. (1977), Theorem 4.13, page 132), $A=\underset{e \in E}{U} A_{e}$ is the expression of $A$ as a semilattice of its archimedean components $A_{e}$. The semigroup $A$ is separative, see the book of Clifford et al. (1977), Theorem 4.13, page 134, $A$ can be embedded in a semigroup $T=U G_{e}$, where $G_{e}$ is a group that contains $A_{e}$.

Assume that $a, b \in A$ and $a \neq b$. Suppose that $a \in$ $A_{e}, b \in A_{b}$. There are two possible cases.

Case 1: $e_{a} \neq e_{b}$. Then $e_{a} e_{b} \neq e_{a}$ or $e_{a} e_{b} \neq e_{b}$.

Without loss of generality, assume that $e_{a} e_{b} \neq e_{a}$.

We create a mapping $\psi: A \rightarrow C^{*}$ in the following way:

$$
\begin{aligned}
& \forall x \in A, \psi(x)= \\
& \left\{\begin{array}{l}
a_{q_{0}} \text { if } x \in A_{e_{x}} \text { and } e_{x} e_{a}=e_{a} ; \\
a_{p_{0}} \text { if } x \in A_{e_{x}} \text { and } e_{x} e_{a} \neq e_{a}
\end{array}\right.
\end{aligned}
$$

where $p_{0}, q_{0}$ are two fixed prime numbers such that $p_{0}>q_{0} \geq 3$.

The mapping $\psi$ is a homomorphism.

Indeed, for any $x \in A_{e_{x}}$ and $y \in A_{e_{y}}$ a/ If $e_{x} e_{a}=e_{a}$ and $e_{y} e_{a}=e_{a}$, then $e_{x y} e_{a}=e_{x} e_{y} e_{a}=$ $e_{x} e_{a}=e_{a}$. It follows that $\psi(x y)=e_{q_{0}}=e_{q_{0}} e_{q_{0}}=$ $\psi(x) \psi(y)$

$\mathrm{b} /$ If $e_{x} e_{a} \neq e_{a}$ and $e_{y} e_{a}=e_{a}$, then $e_{x y} e_{a}=e_{x} e_{y} e_{a}=$ $e_{x} e_{a} \neq e_{a}$. Hence $\psi(x y)=e_{p_{0}}=e_{p_{0}} e_{q_{0}}=\psi(x) \psi(y)$. c/ If $e_{x} e_{a} \neq e_{a}$ and $e_{y} e_{a} \neq e_{a}$, then $e_{x y} e_{a} \neq e_{a}$ (if $e_{a}=$ $e_{x y} e_{a}=e_{x} e_{y} e_{a}=e_{x} e_{x} e_{y} e_{a}=e_{x} e_{x y} e_{a}=e_{x} e_{a} \neq e_{a}$. It is impossible)

We obtain $\psi(x y)=e_{p_{0}}=e_{p_{0}} e_{p_{0}}=\psi(x) \psi(y)$. Thus $\psi$ is a generalized character from $A$ to $C^{*}$, and $\psi(a)=$ $e_{q_{0}} \neq e_{p_{0}}=\psi(b)$.

Case 2. The two elements $a, b$ belong to the same archimedean component $A_{e_{0}}$ of the semigroup $A$.

Because $a \neq b, g=a b^{-1} \neq e_{0}$, where $g \in G_{e_{0}}$ and $G_{e_{0}}$ is a subgroup that contains the semigroup $A_{e_{0}}$.

Assume that $h$ is a height of the element $g$. By the book of Lazlo Fuchs (1967), Lemma 26.4, page 85, there exists a maximum subgroup $H$ of the group $G_{e_{0}}$, such that $G_{e_{0}} / H \approx C\left(q_{0}^{h+1}\right)$ and $g \notin H$.

It follows that there is a homomorphism $\varphi: G_{e_{0}} \rightarrow$ $C\left(q_{0}^{h+1}\right)$ satisfying $\varphi(H)=e_{q_{0}}$ and $\varphi(g) \neq e_{q_{0}}$.

Because $g=a b^{-1}, \varphi\left(a b^{-1}\right) \neq e_{q_{0}} \leftrightarrow$ $\varphi(a) \varphi\left(b^{-1}\right) \neq e_{q_{0}}$.

Finally, $\varphi(a) \neq \varphi(b)$.
Note that $C\left(q_{0}^{h+1}\right)$ is a subgroup of the group $C\left(q_{0}\right)$ and $C\left(q_{0}\right) \approx G_{q_{0}}$, where $G_{q_{0}}$ is a subgroup of the semigroup $C^{*}$.

From this point, we have a generalized character $\varphi$ : $G_{e_{0}} \rightarrow C^{*}$ and $e_{0}=e_{q_{0}}$.

Next, we create a mapping $\psi: T \rightarrow C^{*}$

$$
\begin{aligned}
& \forall x \in T, \psi(x)= \\
& \left\{\begin{array}{l}
e_{p_{0}} \text { if } x \in G_{e_{x}} \text { and } e_{x} e_{0}=e_{0} \\
\psi\left(x e_{0}\right) \text { if } x \in G_{e_{x}} \text { and } e_{x} e_{0} \neq e_{0}
\end{array}\right.
\end{aligned}
$$

where $p_{0}$ is a fixed prime number, such that $p_{0} \neq 3$ and $p_{0}>q_{0}$.

If $x \in G_{e_{x}}$ and $e_{x} e_{0}=e_{0}$, then $x e_{0} \in G_{e_{0}}$.

Direct calculation shows that $\psi$ is a generalized character from the semigroup $T$ into the semigroup $C^{*}$ satisfying the condition $\psi(a)=\varphi(a) \neq \varphi(b)=\psi(b)$. It is obvious that, the mapping $\psi$ is a generalized character from $A$ into $C^{*}$ and this homomorphism separates the two different elements of the semigroup $A$.

Theorem 3.2. Let $X_{2}$ be the class of semigroups $A$ such that $A$ can be embedded into a semilattice of left simple semigroups and $Y_{2}$ be a class of semigroups. If some semigroup from the class $Y_{2}$ contains a semigroup that is isomorphic to the semigroup $C^{*}$, then the class $Y_{2}$ is a class of approximation for the class $X_{2}$ with respect to the Green relation l-equivalency.

Proof. Assume that $Y_{2}$ contains a semigroup $M$ that is isomorphic to the semigroup $C^{*}$.

Let $A$ be a semigroup of the class $X_{2}$. The semigroup $A$ can be embedded into a semilattice of left simple semigroups.

Let $a, b$ be two elements of $A$ such that $a \overline{\mathrm{l}} b$.

Then $b \notin L_{a}$, where $L_{a}$ is $L$ class, that contains the element $a$.

Consider a set $J_{a}=\{x \in A \mid a x \bar{l} a\}$.

Let $c \in J_{a}, d \in A$. Assume that $c d \notin J_{a}$. It is equivalent that acdla.

Because $c d 1 d c, L_{a} L_{c d}=L_{a} L_{d c}$, hence $a c d=a d c$.

From alacd and acdladc, we have aladc. It implies that $a c \mathrm{l}(a d c) c$.

In the other hand, $(a d c) c=(a d)(c c) \in L_{a d} L_{c c} \subset$ $L_{a d} L_{c} \subseteq L_{a d c}$.

And we have got acladc.

Since $a d c l a$, $a c l a$ and $c \notin J_{a}$. It contradicts the assumption.

Thus $c d \in J_{a}$ and it shows that $J_{a}$ is an ideal.

Suppose that $c, d \in A \backslash J_{a}$. Hence $a c \in L_{a}$ and $a d \in L_{a}$. Then $a c d \in L_{a}$. It follows that $c d \notin J_{a}$.

Thus $A \backslash J_{a}$ is a subsemigroup and $J_{a}$ is completely isolated ideal.

Suppose that $a, b \in A$, such that $b \notin L_{a}$. 
Let us consider a mapping $\varphi: A \rightarrow C^{*}$ that is defined in the following way:

$$
\forall x \in A, \varphi(x)=\left\{\begin{array}{l}
e_{q}, \text { if } x \in J_{a} \\
e_{p}, \text { if } x \notin J_{a}
\end{array}\right.
$$

where $p$ and $q$ are two fixed prime numbers such that $q>p \geq 3$.

Due to $J_{a}$ is a completely isolated ideal, $\varphi$ is a generalized character. Moreover $\varphi(a)=e_{p}$ (because $a a \in$ $L_{a}, a \notin J_{a}$ and $\varphi(b)=e_{q}$ ).

Because $b \notin L_{a}, a b \notin L_{a}$.

It is obvious that $e_{p} \bar{l} e_{q}$ in $C^{*}$.

At the end, we obtain $\varphi(a) \bar{l} \varphi(b)$. It means that the semigroup $A$ is approximable with respect to the Green relation l-equivalency.

From the proof of the Theorem 3.2, we have a following result:

Let $C^{* *}=\left\{e_{p} ; e_{q}\right\}$, where $p>q \geq 3$ be a subsemigroup of the semigroup $C^{*}$.

Theorem 3.3. A class of semigroups $Y_{3}$ is a class of approximation for the class $X_{2}$ with respect to the Green relation l-equivalency if and only if some of semigroup from the class $Y_{3}$ is isomorphic to the semigroup $C^{* *}$.

Within the framework of this paper, I only present the results with proof for several number of predicates. Apart from the results for L-equivalences above, I also have found other results with respect to other Green's relations: R-equivalency, D-equivalence and $\mathrm{H}$ - equivalence.

Theorem 3.4. Let $C_{3}$ be the class of semigroups $A$ such that $A$ can be embedded into a semilattice of right simple semigroups. If some of semigroup from a class $S_{3}$ contains a semigroup that is isomorphic to the semigroup $C^{*}$, then the class $S_{3}$ is a class of approximation for the class $S_{3}$ with respect to the Green relation $\Re$-equivalency.

\section{CONCLUSION}

One of the important directions in modern algebra is the study of not only the algebraic system itself, but also the systems derived from it. Using the relationships established in the paper, it is possible to extend the obtained results to other classes of algebraic structures, other sets of homomorphisms and other predicates.

Finding a minimal class of approximation for a wellknown class of structures with respect to important predicates is extremely sophisticated. Vinh et al. (2018) have proved that the semigroup $C^{*}$ is a minimal semigroup of approximation for the class $C_{4}$ of commutative, regular and periodic semigroups with respect to the predicate $P$ of the possible of belonging an element to a subsemigroup and in the book of
Korabelsh'chikova et al. (2013), the authors described another semigroup that played a role as a minimal semigroup of approximation for the class $C_{4}$ with respect to $P$. In order to find a class of approximation for the class $C_{4}$ we have to clarify common features between these two semigroups.

\section{ACKNOWLEDGEMENT}

This research is funded by Vietnam National University Ho Chi Minh City (VNU-HCM) under grant number C2019-20-03

\section{CONFLICT OF INTERESTS}

The author declares that there is no conflict of interests regarding the publishing of this paper.

\section{AUTHORS' CONTRIBUTIONS}

Dang Van Vinh is in charge of all research content presented in the article.

\section{REFERENCES}

1. Dang VV, Korabelsh'chikova SY, Mel'nicov BF. SH - weak duality of semigroups and minimum semigroup of $\mathrm{SH}$ - approximation. Izvestia Vushix Uchevnux Zavedenhia, UDK 512.53, 510.54. 2019;Available from: 10.21685/2072-3040-2019-1-3; https://izvuz_fmn.pnzgu.ru/fmn3119.

2. Dang VV, Korabelsh'chikova SY, Mel'nicov BF. Minimal $\mathrm{SH}$-approximation of semigroups. Algebras and Lattices in Hawai'i , Honolulu, United States. 2018;p. 31-38. Available from: https://universalalgebra.github.io/ALH-2018/assets/ ALH-2018-proceedings-6x9.pdf.

3. Dang VV, Korabelsh'chikova SY, Mel'nicov BF. Semigroups approximation with respect to some ad hoc predicates. Arctic environmental research. 2017;17:133-140. Available from: https://doi.org/10.17238/issn2541-8416.2017.17.2.133.

4. Dang VV, Korabelsh'chikova SY, Mel'nicov BF. Some issues of semigroup approximations. Izvestia Vushix Uchevnux Zavedenhia. Russia. 2017;p. 46-57. Available from: https://cyberleninka.ru/article/v/nekotorye-voprosyapproksimatsii-polugrupp.

5. Dang VV, Korabelsh'chikova SY, Mel'nicov BF. On the problem of finding minimum semigroup of approximation. Izvestiya Vysshikh Uchebnykh Zavedenhii, Povolzhskiy Region. Russia. 2015;p. 88-98. Available from: http://izvuz_fmn.pnzgu.ru/ sf7315.

6. Lesokhin MM. On the approximation of semigroups with respect to the predicate. Uch. Zap. of Herzen LGPI. Russia . 1971;404:191-219.

7. Lesokhin MM, Golubov EA. The finite approximability of commutative semigroups. Matem. Zap. Uralskogo Un-ta. Russia. 1966;5(3):82-90.

8. Ignatyeva IV. SH approximation of semigroup by finite characters. Modern algebra (Interuniversity collection of scientific papers), V.1 (21), Rostov on Don, Russia. 1996;p. 25-30.

9. Golubov EA. The finite approximability of separable, naturally linearly ordered commutative semigroups. Izv. Vuzov, Matem. 1969;(2):j23-31.

10. Kublanovskiy SI. On the residual finiteness prevarieties semigroups with respect to the predicate. Modern algebra, Groupoids and their homomorphisms. - Leningrad. 1980;p. 58-88.

11. Fuchs L. Abelian groups. Pergamon Press Ltdl, Headington Hill Hall, Oxford. 1967;. 
12. Mamikonyan SG. Varieties of finitely approximate semigroups. Matem.Sb. Russia. 1972;88(3):353-359. Available from: https://doi.org/10.1070/SM1972v017n03ABEH001509.

13. Tutygin AG, Yashina EY. The dependence of conditions of approximation semigroups with respect to various predicates. Modern algebra (Interuniversity collec-tion of scientific papers), V.3 (23), Rostov on Don, Russia. 1998;p. 136-141.

14. Zyabliceva LV, Korabelshchikova SY, Popov IN. Some special semigroups and their homomorphisms. Arkhangelsk, Pomor
State University, Russia. 2013;

15. Dang VV, Korabelsh'chikova SY, Mel'nicov BF. Class of approximation. Izvestia Vushix Uchevnux Zavedenhia, UDK 512.53 510.54, Russia. 2019;Available from: https://izvuz_fmn.pnzgu. $\mathrm{ru} / \mathrm{fmn} 5419$.

16. Clifford AH, Preston GB. The Algebraic Theory of Semigroups. Volume 1, the American Mathematical Society. 1977; 